\title{
Recomendaciones para el manejo de pacientes con COVID-19 con indicación terapéutica de ventilación mecánica que eventualmente son conectados a máquinas de anestesia
}

\author{
Aranda F $\mathrm{F}^{1}$, Aliste $\mathrm{J}^{2}$, Altermatt $\mathrm{F}^{3}$, Alvarez JP, Bernucci $\mathrm{F}^{4}$, Bruhn $\mathrm{A}^{3}$, Cabrera MC 1 , \\ Carrasco $E^{5}$, Castillo $R^{6}$, De la Fuente $\mathrm{R}^{3}$, Díaz $\mathrm{R}^{6}$, Egaña $\mathrm{Jl}^{2}$, González $\mathrm{R}^{2}$, Honorato $T^{6}$, \\ Lacassie $\mathrm{HJ}^{3}$, López $\mathrm{M}^{6}$, Merino $\mathrm{W}^{7}$, Penna $\mathrm{A}^{2}$, Pizarro $\mathrm{F}^{6}$, Torres $\mathrm{D}^{8}$, Cristián Rocco ${ }^{6}$, \\ Alejandro Bruhn ${ }^{3}$, Darwin Acuña ${ }^{9}$, Tomás Regueira ${ }^{6}$
}

\section{Lista de abreviaturas}

\begin{tabular}{|l|l|}
\hline APL & Adjustable pressure limiting valve \\
\hline APSF & Anesthesia Patient Safety Foundation \\
\hline ASA & American Society of Anesthesiologists \\
\hline COVID-19 & $\begin{array}{l}\text { Enfermedad por Coronavirus 2019- } \\
\text { nCoV o SARS-CoV-2 }\end{array}$ \\
\hline EPP & Equipos de protección personal \\
\hline FDA & Food and Drug Administration \\
\hline FGF & Flujo de gases frescos \\
\hline HMEF & $\begin{array}{l}\text { Filtro intercambiador de humedad y } \\
\text { calor }\end{array}$ \\
\hline MA: & Máquina de anestesia. \\
\hline Relación I:E & Relación inspiración:espiración \\
\hline SACH & Sociedad de Anestesiología de Chile \\
\hline SDRA & $\begin{array}{l}\text { Síndrome de distress respiratorio del } \\
\text { adulto }\end{array}$ \\
\hline UCI & Unidad de cuidados intensivos \\
\hline VCI & Ventilador de cuidados intensivos \\
\hline VM & Ventilación mecánica. \\
\hline
\end{tabular}

\section{Desarrollo de la RC}

\subsection{Introducción}

a pandemia de COVID-19 producida por SARSCoV-2 actualmente en curso anticipa una gran demanda por ventiladores mecánicos (VM), ya que un porcentaje relevante de los contagiados cae rápidamente en insuficiencia respiratoria y requiere de cuidados intensivos. Anticipándose a ese exceso de demanda y considerando que es muy probable que el número actual de ventiladores mecánicos en las unidades de cuidados intensivos (UCI) sean insuficientes, se ha solicitado a la SACH un informe técnico en relación al uso de las máquinas de anestesia como VM.

Chile no es el primer país que se ve enfrentado a esta situación. La FDA ha aprobado temporalmente el uso de máquinas de anestesia como ventiladores y la ASA junto con la APSF han publicado una declaración conjunta donde se reconoce que "las máquinas de anestesia no están diseñadas para entregar apoyo ventilatorio a largo plazo, sin embargo, se transforman en la primera línea natural de apoyo durante la pandemia cuando no hay suficientes ventiladores de cuidados intensivos (VCI) en UCI para el cuidado de los pacientes" $[1,2]$.

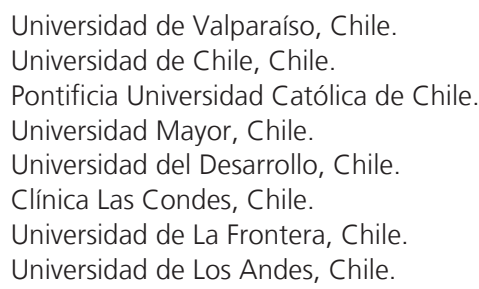


En el contexto de un conflicto ético entre la buena práctica clínica y los recursos disponibles para enfrentar la crisis, la SACH decide redactar estas recomendaciones, en el entendido de que es una situación extraordinaria que empuja a la utilización de un recurso físico, la máquina de anestesia (MA), que no está diseñada para VM de pacientes con SDRA y que no cuenta con las herramientas mecánicas, dispositivos y alarmas necesarias para un correcto manejo. Además, el recurso profesional (especialista) no está familiarizado necesariamente con este tipo de pacientes de acuerdo a su desempeño habitual y puede no contar con las necesarias actualizaciones y experticia que corresponden al ámbito específico de una sub-especialidad como lo es la de medicina intensiva, con las complejidades y consecuencias que esto conlleva. Así, es necesario estandarizar el manejo de MA a través de una recomendación para que puedan ser utilizadas en la forma más segura y efectiva posible, considerando que el uso de las MA como VM "es una maniobra de salvataje" $[3,4]$.

Con estas recomendaciones, la SACH pretende entregar orientaciones para enfrentar un paciente que es conectado a VM por un cuadro positivo para COVID-19 utilizando una MA, ante la imposibilidad de disponer de un VCI. Así, la SACH cumple con su propósito, su responsabilidad ética y compromiso con el país en el contexto de esta crisis global.

\subsection{Objetivo General}

Recomendar cómo enfrentar un paciente que es conectado a VM por un cuadro positivo para COVID-19 utilizando MA ante la imposibilidad de obtener un VCl estándar.

\subsection{Escenarios clínicos o ámbito de aplicación de la recomendación}

Esta recomendación se aplica solamente en el contexto del manejo de un paciente con diagnóstico de infección por COVID-19 que, al no existir otro recurso, es necesario conectar a VM utilizando una MA.

\section{Métodos}

\subsection{Métodos de consenso utilizados para la for- mulación de recomendaciones}

En la formulación de la recomendación clínica (RC) se establecieron controversias con respecto a:

- La condiciones de aplicabilidad o el impacto esperado de las recomendaciones en el contexto de la pandemia.
- Las publicaciones encontradas resulten contradictorias o las condiciones locales no hagan aplicable la recomendación.

Para resolver estas diferencias aplicaron métodos de consenso:

- Representatividad: Todos los autores o al menos tres de ellos participaron de la resolución de la controversia.

- Todos los miembros del grupo a cargo de la elaboración del documento dispusieron del mismo nivel de información básica para la toma de decisiones.

\subsection{Grados de evidencia y niveles de recomen- dación utilizados en la RC}

Para esta recomendación la evidencia disponible descansa principalmente en opinión de expertos.

Según la evolución epidemiología de la pandemia y la aparición de nueva evidencia se realizará actualizaciones de este documento según se necesario.

\subsection{Consideraciones ético-sociales}

A través de este documento, la SACH desea entregar una herramienta que permita, a profesionales y autoridades, enfrentar de mejor manera a pacientes con diagnóstico de COVID-19 que requieren VM cuya única alternativa, por la escasez de recursos, es conectarlo a una MA. Se pone a disposición de los pacientes, autoridades sanitarias y anestesiólogos(as) del país, información pertinente para confrontar los nuevos desafíos que esta pandemia nos presenta a todos.

\section{Recomendaciones}

\subsection{Adecuación y mantención de la MA para VM en pacientes con COVID-19}

\subsubsection{Generalidades}

Las MA tienen un principio de funcionamiento y una interfaz de usuario diferente que los $\mathrm{VCl}$.

A diferencia de los $\mathrm{VCl}$, las MA tienen un circuito interno que permite reutilizar parcialmente los gases espirados por el paciente, con el objetivo de ahorrar gases anestésicos, disminuir la contaminación, conservar la humedad y temperatura. Esto permite utilizar flujos de gases frescos (FGF) muy inferiores al volumen minuto del paciente, lo que se conoce como circuito semicerrado. Los VCI son circuitos abiertos.

Una de las principales consecuencias de un sistema semicerrado (como las MA) es que produce rein- 
halación de gases. Por esto, dado que el aire espirado tiene un alto contenido de $\mathrm{CO}_{2}$, se utiliza un absorbedor de $\mathrm{CO}_{2}$ (cal sodada), en serie en el circuito. El recambio de los gases dentro del circuito depende del flujo total de gases frescos y la $\mathrm{FiO}_{2}$ dependerá de la relación entre el oxígeno y aire administrado. La racionalidad de usar FGF bajos en anestesia es principalmente ahorrar gases anestésicos y disminuir la contaminación secundaria. Sin embargo, si se usan MA para tratar pacientes críticos, el uso de gases anestésicos no se recomienda y por tanto la ventaja de usar bajos FGF desaparece.

Todas las MA permiten utilizar modo manual/ espontáneo, que es un modo único no presente en la mayoría de los VCl. Mediante un interruptor o un botón en la consola, permite ventilar con la bolsa reservorio, regulando la presión máxima mediante la válvula adjustable pressure limiting (APL). La válvula APL sólo está activa en modo manual/espontáneo y no tiene influencia en la VM. Si llevamos la válvula APL a $30 \mathrm{~cm}$ de $\mathrm{H}_{2} \mathrm{O}$ podremos ventilar a un paciente hasta esa presión, pero también progresivamente se alcanzará esa presión por la entrada de FGF en el circuito, pudiendo ser deletéreo para el paciente. Dejada en $0 \mathrm{~cm}$ de $\mathrm{H}_{2} \mathrm{O}$, el paciente tendrá la menor resistencia en caso de ventilar espontáneamente, lo que no es deseable.

Los ventiladores de las MA mayormente usados son el de fuelle accionado por gas comprimido (Ej. Datex Ohmeda) y el de pistón accionado por un mecanismo eléctrico (Ej. Dräger). Los últimos modelos de MA tienen versiones de ventiladores más sofisticados que podrían integrar funciones símiles a los $\mathrm{VCl}$, lo que no implica que tengan desempeño similar. Por lo general traen modos ventilatorios básicos $(\mathrm{VC}, \mathrm{PC}$, PS, SIMV). Los ventiladores más adecuados son los de pistón o turbina (mayor precisión), seguidos de los de fuelle descendente con compensación por compliance y de FGF.

Las MA no tienen mecanismo para compensar fugas, en caso de existir, la PEEP podría no mantenerse y la ventilación se verá afectada, lo cual se verá reflejado por una alarma de fuga. Por esta razón no son aptos para ventilación no invasiva.

El capnógrafo es de muestreo lateral, no central, lo que implica que existe un retardo en la monitorización del $\mathrm{CO}_{2}$ y genera acumulación de vapor de agua. Para evitar que el gas aspirado por capnografía se contamine, se recomienda usarlo siempre entre el filtro HEM y la máquina.

La distribución de alarmas a través de la interfaz en serie no está diseñada de manera redundante (a prueba de fallas). Por lo tanto, una supervisión remo- ta, en la que un usuario no está cerca del dispositivo, debe asegurarse de que el volumen de la alarma esté configurado al máximo posible para aumentar la probabilidad de que las situaciones potencialmente peligrosas se reconozcan a tiempo. Particularmente importantes en estos temas son los límites de alarma para el volumen minuto (límite inferior y superior) y el $\mathrm{CO}_{2}$ espiratorio (límite inferior y superior).

Finalmente, destacamos lo altamente riesgoso de conectar dos o más pacientes a una MA con alto riesgo evidente de hipoventilación y barotrauma simultáneos.

\subsubsection{Decisión de conectar un paciente con COVID-19 a una MA}

1. Recomendamos, cuando sea necesario, conectar a VM a un paciente a una MA únicamente en caso de no existir otro recurso de apoyo ventilatorio.

2. Recomendamos fuertemente la presencia de un anestesiólogo para el manejo de la MA. El(la) anestesiólogo(a) es el responsable del manejo ventilatorio, quien eventualmente puede delegar el cuidado del paciente a otro anestesiólogo(a), a un(a) residente de anestesiología, a un(a) enfermero(a) o a un(a) técnico(a) de anestesia que esté debidamente entrenado(a) para la supervisión del funcionamiento de la MA[5].

3. Recomendamos fuertemente el manejo médico del paciente conectado a una MA por infección por COVID-19 por un médico intensivista. NO recomendamos conectar a un paciente a una MA como $\mathrm{VCl}$ si no se cuenta con manejo médico por un intensivista.

4. NO recomendamos el uso de MA como VM por profesionales no anestesiólogos.

5. La SACH recomienda fuertemente que se ocupen todos los EPP indicados cuando se realicen procedimientos de alto riesgo como intubación traqueal y manipulación de pacientes conectados a ventilación mecánica[6].

6. Recomendamos fuertemente que las instituciones elaboren un plan sobre quién y cómo proporcionará atención médica a estos pacientes[7].

\subsubsection{Antes de conectar un paciente a la $M A$}

1. Recomendamos $N O$ considerar $V M$ en recintos que no cuenten $\mathrm{O}_{2}$ y aire (aire comprimido) como gases clínicos.

2. El(la) anestesiólogo(a) debe estar familiarizado(a) con la MA que va a usar como VM.

3. El manual de la MA debe estar disponible.

4. Recomendamos no utilizar la mesa quirúrgica como cama del paciente conectado a VM. 
5. Antes de conectar a un paciente, se debe realizar la verificación habitual del estado correcto del dispositivo o MA. Asegurar que todos los accesorios como: mangueras de ventilación, filtro de bacterias, línea de muestreo de gases, sensor de oxígeno en línea, bolsa de respiración manual, estado del absorvedor de $\mathrm{CO}_{2}$ y trampas de agua, estén conectados correctamente y que el dispositivo pueda generar flujo de gas y presión en el conector del paciente.

6. Siempre debe haber una bolsa autoinflable manual (Ambu ${ }^{\circledR}$ o similar) disponible que permita la ventilación de respaldo del paciente en caso de problemas o mal funcionamiento de la MA.

7. En fase aguda el paciente se mantendrá sedado y en modo ventilatorio controlado. Los requerimientos mínimos de la MA son que el ventilador tenga modo volumen control, sensor de flujo para calcular el volumen corriente y PEEP de al menos $20 \mathrm{cmH}_{2} \mathrm{O}$.

8. Idealmente utilizar MA que cuenten además con modos PC y PS, que tenga posibilidad de programar pausa inspiratoria, que tenga curvas de presión de vía aérea, de flujo y que entregue los valores de presión pico, presión meseta y presión media. Modelos frecuentes en nuestro medio tales como Draeger Fabius GS o GE Datex Ohmeda Aestiva 5-7900 cumplen estos requisitos.

9. NO recomendamos utilizar MA más antiguas tales como GE Datex Ohmeda Excel 210-7800 porque carecen de mecanismos de compensación, por lo cual no deben usarse para ventilar pacientes críticos (Tabla 1).

10. NO recomendamos utilizar MA para ventilación no invasiva.

11. Recomendamos usar la toma de muestra de capnografía siempre entre el filtro (HEMF, con capacidad de filtrado viral) y la máquina.

12. Recomendamos el volumen de las alarmas ajustados lo suficientemente alto para escucharse a la distancia en que está el operador, especialmente en entornos ruidosos.

13. Recomendamos una prueba del sistema cada 24 horas.

14. Verificar regularmente: absorbedor de $\mathrm{CO}_{2}$ agotado, disponibilidad de cal sodada, trampa de agua llena, agua estancada en las mangueras de respiración y condensación excesiva en el filtro que puede conducir a una mayor resistencia y producir una hipoventilación de los pacientes.

15. Recomendamos la remoción de los vaporizadores y la desconexión de óxido nitroso.

16. Recomendamos conexiones de aire y oxígeno a alta presión junto con balones de reserva. Se recomienda 2 balones.

17. Recomendamos que la concentración inspirada de oxígeno debe ser monitorizada en todo momento, con las alarmas necesarias para detectar concentraciones menores a las programadas y realizar los ajustes necesarios.

18. NO recomendamos la administración de anestesia inhalatoria para sedación de pacientes ventilados.

19. NO recomendamos utilizar la MA en modo manual.

20. NO recomendamos conectar dos o más pacientes a una MA.

21. Recomendamos informar a Equipos Médicos del hospital que se ocupará una o varias MA para VM prolongada, con el fin de tener una asistencia oportuna y asegurar la disponibilidad de los insumos asociados y repuestos.

\section{Información:}

Como la prueba del sistema dura hasta ocho minutos (dependiendo del tipo de dispositivo), se requiere la asistencia de un usuario experimentado para este paso y apoyo con la ventilación manual o asistida del paciente (recuerde clampear tubo si desconecta circuito, tener mucho cuidado de aplicar presiones limitadas, y minimizar aerosoles).

Dräger $^{\circledR}$ informa que si una prueba del sistema cada 24 horas no es factible debido a razones clínicas, recomienda realizar la prueba al menos cada 72 horas para reducir la probabilidad de mal funcionamiento del dispositivo.

Datex Ohmeda ${ }^{\circledR}$ informa que sus equipos se apagan luego de funcionar 49 días consecutivos sin reiniciarse. La prueba de sistema demora menos de 10 minutos.

La FDA sugiere utilización de los insumos hasta evidencias de malfuncionamiento o suciedad para favorecer su duración y evitar agotamiento de suministros.

\subsection{Protocolo de ventilación mecánica en máqui- na de anestesia}

\subsubsection{Generalidades}

El manejo de la MA como VM en pacientes COVID-19 es de responsabilidad de un(a) anestesiólogo(a).

Debe existir un protocolo de chequeo y eventual ajuste de los parámetros de VM y mantención de la MA previamente establecido. 


\begin{tabular}{|c|c|c|c|c|c|c|c|}
\hline $\begin{array}{l}\text { Modelo máquina } \\
\text { anestesia }\end{array}$ & $\begin{array}{l}\text { Drive del } \\
\text { ventilador }\end{array}$ & Pmax & $\begin{array}{l}\text { FR max } \\
\text { (resp/min) }\end{array}$ & $\begin{array}{l}\text { PEEP max } \\
\left(\mathrm{cmH}_{2} \mathrm{O}\right)\end{array}$ & $\begin{array}{l}\text { Vt (ml) / } \\
\text { MV max } \\
(\mathrm{L} / \mathrm{min})\end{array}$ & $\begin{array}{c}\text { Compensación } \\
\text { Compliance/ } \\
\text { Sensor flujo }\end{array}$ & Observación \\
\hline Draeger Apollo & E - Pistón & 70 & 100 & 20 & $1.400 / 50$ & Sí / Sí & \\
\hline Draeger Fabius GS & E - Pistón & 70 & 60 & 20 & $1.400 / 100$ & Sí / Sí & \\
\hline Draeger Perseus & E - Turbina & 80 & 100 & 35 & $1.500 / 40$ & Sí /Sí & \\
\hline GE Aisys & P - Fuelle & 100 & 100 & 30 & $1.500 / 120$ & Sí / Sí & \\
\hline GE Aisys C2 & P - Fuelle & 100 & 100 & 30 & $1.500 / 120$ & Sí /Sí & \\
\hline GE Avance & P - Fuelle & 100 & 100 & 30 & $1.500 / 120$ & Sí / Sí & \\
\hline GE Avance C2 & P - Fuelle & 100 & 100 & 30 & $1.500 / 120$ & Sí / Sí & \\
\hline $\begin{array}{l}\text { GE Carestation } \\
600 \text { series }\end{array}$ & P - Fuelle & 100 & 100 & 30 & $1.500 / 100$ & Sí / Sí & \\
\hline $\begin{array}{l}\text { GE (Datex Ohmeda) Aestiva } \\
5 \text { - Ventilator } 7900\end{array}$ & P- Fuelle & 100 & 100 & 30 & $1.500 / 100$ & Sí / Sí & \\
\hline $\begin{array}{l}\text { GE (Datex Ohmeda) } \\
\text { Excel 210-7800 }\end{array}$ & $P$ - Fuelle & 100 & 100 & No & $1.500 / 150$ & $\mathrm{No} / \mathrm{No}$ & $\begin{array}{l}\text { No recomendada } \\
\text { para paciente } \\
\text { crítico }\end{array}$ \\
\hline Getinge Flow-i & P - Reflector & 80 & 100 & 50 & & Sí / Sí & \\
\hline Mindray A7 Advantage & P - Fuelle & 100 & 100 & 30 & $1.500 / 30$ & Sí / Sí & \\
\hline Mindray A5 Advantage & P - Fuelle & 100 & 100 & 30 & $1.500 / 30$ & Sí / Sí & \\
\hline Mindray A4 Advantage & P - Fuelle & 100 & 100 & 30 & $1.500 / 30$ & Sí / Sí & \\
\hline
\end{tabular}

E: Electrónico. P: Pneumático.

Minimizar las desconexiones del circuito, para proteger al personal de salud y pacientes frente a la posibilidad de generación de aerosoles.

Todo paciente que va a ser sometido a VM invasiva es un paciente grave. La recomendación es realizar una intubación traqueal precoz.

Si tiene un aumento rápido de presiones de vía aérea con hipoventilación: considere como primera maniobra cambiar el filtro de vía aérea

\subsubsection{Estrategia}

\section{Circuito abierto vs circuito cerrado}

Dentro de las ventajas de usar un flujo de gases frescos (FGF) alto se cuentan:

- Administrar una $\mathrm{FlO}_{2}$ segura y constante.

- Evitar cambios reiterados de absorbentes de $\mathrm{CO}_{2}$ (cal sodada) con la consecuente protección del personal de salud y ahorro en EPP.

- Evitar humidificación de circuito, válvulas y sensores lo que aumenta el riesgo de mal funcionamiento.
- La presencia de un filtro HEM es suficiente para entregar gases adecuadamente humidificados al paciente. Esta es una de las razones por las cuales el filtro HME es indispensable al usar una MA como VM.

- Uno de los inconvenientes de los FGF altos es el mayor consumo de $\mathrm{O}_{2}$ y aire desde la red.

Información: El uso de FGF bajos ( circuito cerrado) posee la ventaja de generar un potencial ahorro de gases medicinales $\left(\mathrm{O}_{2}\right.$ y aire), pero posee desventajas como: riesgo de una desigualdad entre la $\mathrm{FiO}_{2}$ seleccionada y la verdaderamente administrada, requerir una monitorización más estrecha de la mezcla de gases, menor capacidad de compensar eventuales fugas, aperturas frecuentes del circuito (cambio de absorbedor de $\mathrm{CO}_{2}$ ) con el consecuente riesgo de contaminación del ambiente/personal, mayor consumo de absorbedor, eventual humidificación y mal funcionamiento de los componentes del circuito (válvulas, líneas de muestreo, etc.) entre otras. 
Recomendamos: “Usar un FGF similar o 20\% superior al volumen minuto del paciente, con lo cual la MA pasa a funcionar como sistema abierto".

\section{Volumen corriente (Vt)}

Información: El cálculo del peso ideal se debe realizar con las siguiente fórmula (fórmula ARDSNet):

a) Si es hombre: $50+[0,91 \times$ (altura en $\mathrm{cm}$ )-152,4].

b) Si es mujer: $45,5+[0,91 \times$ (altura en $\mathrm{cm}$ )-152,4]. Si presión meseta $>30 \mathrm{cmH}_{2} \mathrm{O}$, disminuir Vt de a $1 \mathrm{ml} / \mathrm{kg}$ hasta un mínimo de $4 \mathrm{ml} / \mathrm{kg}$.

Hipercapnia o hipoxemia: aumentar Vt de $1 \mathrm{ml} / \mathrm{kg}$ hasta $8 \mathrm{ml} / \mathrm{kg}$ como máximo, manteniendo presiones protectoras (ver presiones de vía aérea).

Recomendamos: Una ventilación con 4 a $8 \mathrm{ml} / \mathrm{kg}$ de peso ideal con el fin de minimizar el daño pulmonar asociado a ventilación mecánica.

\section{PEEP (Presión positiva al final de espiración)}

Información: La siguiente tabla de combinación $\mathrm{FiO}_{2} /$ PEEP puede ser utilizada como referencia apuntando a una meta de oxigenación de $\mathrm{SpO}_{2} 90-96 \%$, o $\mathrm{PaO}_{2} 60-80 \mathrm{mmHg}$.

\begin{tabular}{|l|l|l|l|l|l|l|l|l|l|l|l|l|l|l|l|l|l|}
\hline $\mathrm{FiO}_{2}$ & 0,3 & 0,4 & 0,4 & 0,5 & 0,5 & 0,6 & 0,7 & 0,7 & 0,7 & 0,8 & 0,9 & 0,9 & 0,9 & 1 & 1 & 1 & 1 \\
\hline PEEP & 5 & 5 & 8 & 8 & 10 & 10 & 10 & 12 & 14 & 14 & 14 & 16 & 18 & 18 & 20 & 22 & 24 \\
\hline
\end{tabular}

Algunos pacientes podrían requerir una estrategia de PEEP alta (ver 5.2.2.10) pero se sugiere reservar esa opción sólo para pacientes que persistan con PaFi $<150$ después de implementar medidas como posición prono y bloqueo neuromuscular.

Recomendamos: Usar inicialmente una estrategia de PEEP moderada en adultos conectados a VM con COVID-19.

\section{PEEP Presiones de vía aérea*}

Información: Es fundamental vigilar el valor de la presión meseta (refleja presión alveolar de fin de inspiración), y la presión de distensión o driving pressure (corresponde a la diferencia de presión entre $\mathrm{P}$ meseta y PEEP, y refleja la variación de presión cíclica a nivel alveolar). Si bien en muchos protocolos se recomienda medir la presión meseta con una pausa prolongada ( $>0,5 \mathrm{seg}$ ), en términos prácticos esto no es necesario siendo suficiente la presión meseta que mide el ventilador ciclo a ciclo al programar una pausa de $\pm 30 \%$ del tiempo inspiratorio (equivalente a aproximadamente 0,2 a 0,5 seg).

Recomendamos: Dirigir la presión de meseta o presión plateau (Pplat) a valores $\leq 28 \mathrm{~cm} \mathrm{H}_{2} \mathrm{O}$ en adultos conectados a VM con COVID-19 y SDRA.

Recomendamos: Dirigir la presión de distensión $(\Delta P)$ (en inglés driving pressure) a valores $\leq 14 \mathrm{~cm}$ $\mathrm{H}_{2} \mathrm{O}$ en adultos conectados a VM con COVID-19 y SDRA.

\section{Frecuencia respiratoria (Fr)}

Recomendamos: ajustar la frecuencia respiratoria necesaria para mantener una $\mathrm{PaCO}_{2}$ entre 35 y 60 $\mathrm{mmHg}$ (manteniendo un pH mayor a 7,25).

Información: Normalmente estos pacientes requerirán frecuencias de 20 a 30 respiraciones /min para lograr estas metas.

\section{Fracción inspirada de oxígeno $\left(\mathrm{FiO}_{2}\right)$}

Recomendamos: Una $\mathrm{FiO}_{2}$ necesaria para lograr saturación de $\mathrm{O}_{2}$ entre $90 \%$ y $96 \%$.

Recomendamos: $\mathrm{UNa} \mathrm{FiO}_{2}$ necesaria para lograr presión parcial de oxígeno arterial $\left(\mathrm{PaO}_{2}\right)$ mayor a 60 $\mathrm{mmHg}$.

\section{Maniobras de reclutamiento}

Información: Las maniobras de reclutamiento pueden mejorar transitoriamente la oxigenación. Sin embargo, su aplicación puede generar alteraciones hemodinámicas y respiratorias importantes. Por lo tanto se recomienda reservarlas para situaciones de hipoxemia severa puntuales generadas por desconexiones accidentales del VM, o bien considerar su uso al implementar una estrategia de PEEP alta. Antes de realizarlas deben ajustarse los parámetros ventilatorios y ver si existe mejora.

Para adultos con ventilación mecánica con COVID-19 e hipoxemia a pesar de optimizar la ventilación, sugerimos usar maniobras de reclutamiento, en lugar de no usar reclutamiento maniobras.

Recomendamos: Si ha decidido usar maniobras de reclutamiento, recomendamos realizar una maniobras de reclutamiento progresiva manteniendo ventilación cíclica y llegando a una presión inspiratoria máxima de $40 \mathrm{cmH}_{2} \mathrm{O}$, en lugar de una maniobra de inspiración mantenida, la cual se asocia a más complicaciones. 


\title{
Uso de MA como VM en pacientes Covid-19
}

\author{
Utilizar una MA en vez de un \\ VM sólo como último recurso
}

Para conectar un paciente Covid-19 a una MA:

- La MA debe ser manejada por un(a) anestesiólogo(a)

- El manejo médico debe ser por intensivista

- Se deben utilizar siempre los EPP

- NO recomendamos su uso por no-anestesiólogos

- Solo frente a necesidad se entrenará a personal

diferente al anestesiólogo para su manejo
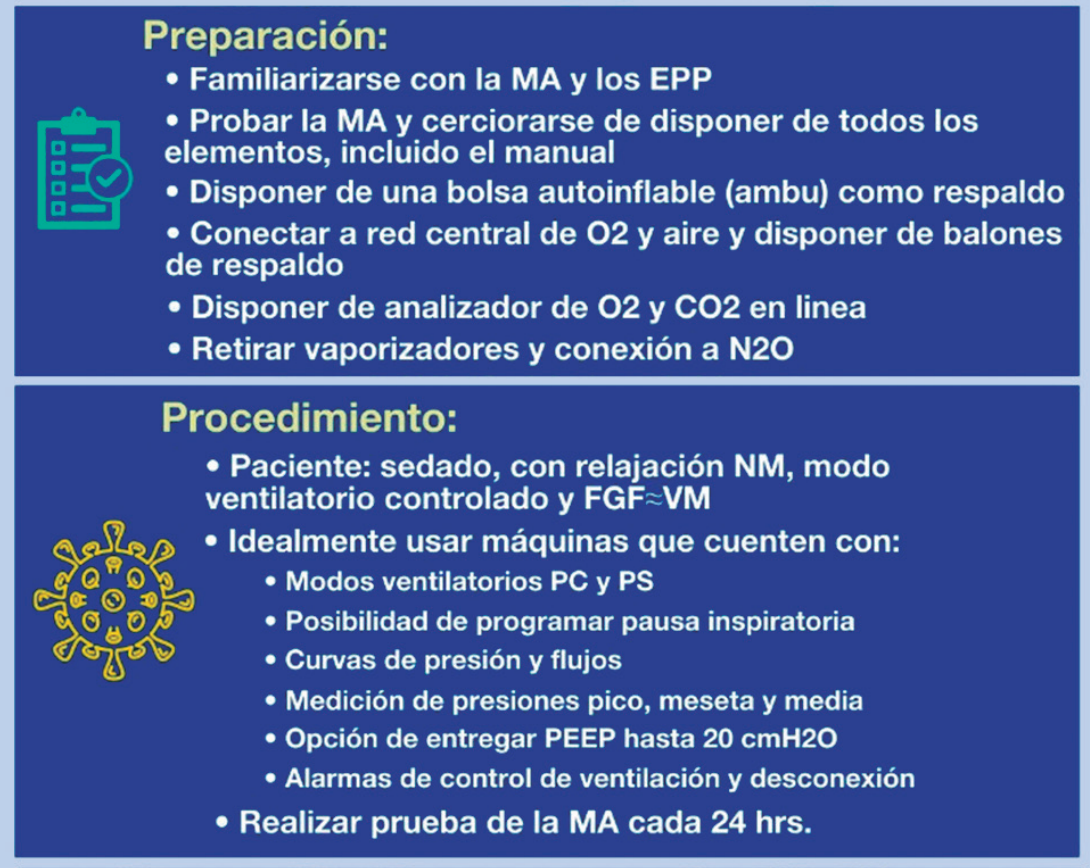

Precaución (riesgoso para el paciente):

- NO recomendamos la anestesia inhalatoria para sedación

- NO recomendamos conectar 2 o más pacientes a una MA

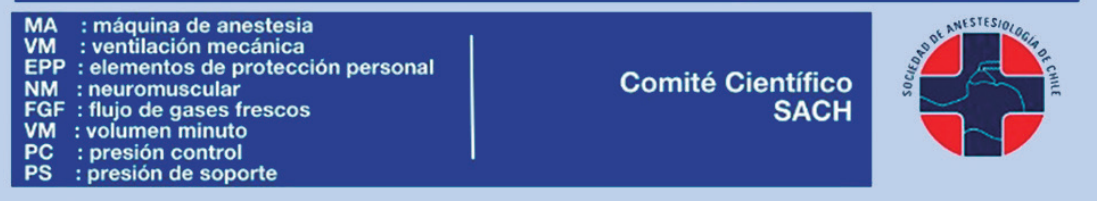

Figura 1. Infografía. 


\section{Posición prono}

Información: Esta estrategia ha demostrado disminuir la mortalidad en SDRA y mejorar sustantivamente la oxigenación en la mayoría de los pacientes, y la experiencia internacional reportada en casos de COVID-19 es muy favorable.

Recomendamos: Utilizar posición prono en aquellos pacientes que habiéndose aplicado una ventilación inicial con volumen corriente bajo y PEEP moderado persistan con $\mathrm{PaFi}<150$, o si se requiere PEEP > $14 \mathrm{cmH}_{2} \mathrm{O}$ para mantener este nivel de PaFi. Si no existe experiencia en su uso se recomienda seguir el protocolo y guía publicada en sitio web SOCHIMI (si quieren pueden pedir a SOCHIMI subirla a web SACH), y/o solicitar asistencia de profesionales de intensivo.

\section{Bloquedares neuromusculares en infusión continua}

Recomendamos: Utilizar bloqueadores neuromusculares en infusión continua al menos durante las primeras 48 horas en aquellos pacientes que habiéndose aplicado una ventilación inicial con volumen corriente bajo y PEEP moderado persistan con PaFi $<150$, o si se requiere PEEP $>14 \mathrm{cmH}_{2} \mathrm{O}$ para mantener este nivel de PaFi, así como en pacientes en posición prono o en aquellos que desarrollen disincronías frecuentes paciente-ventilador.

\section{Estrategia de PEEP alta}

Información: Las estrategia de PEEP alto han mostrado mejorar la oxigenación en la mayoría de los pacientes pero no se ha demostrado que mejoren desenlaces y pueden generar complicaciones. Una estrategia de PEEP alta requiere vigilancia más estricta por riesgo de compromiso hemodinámico o sobre- distensión pulmonar. Se sugiere la siguiente tabla de $\mathrm{FiO}_{2} /$ PEEP del protocolo Lung Open Ventilation

Se debe evaluar que el nivel de PEEP programado no esté generando sobredistensión. Signos sugerentes de sobredistensión posterior a un aumento en nivel de PEEP son:

a) Disminución de compliance (compliance $=$ volumen corriente / (presión meseta-PEEP). Refleja predominio de sobredistensión por sobre reclutamiento.

b) Compromiso hemodinámico significativo (refleja disminución retorno venoso o disfunción ventrículo derecho).

c) Aumento en $\mathrm{PaCO}_{2}$ (refleja aumento espacio muerto).

Recomendamos: Reservar esta estrategia para pacientes que persistan con PaFi $<150$ a pesar de medidas previas.

Recomendamos: Consultar antes a médico intensivista para evaluar riesgos y beneficios.

Recomendamos: SI sospecha sobredistensión, disminuir nivel de PEEP y consultar a intensivista.

\section{Sistemas de aspiración de secreciones.}

Recomendamos: Tener un sistema de aspiración de secreciones cerrado con el fin de evitar la aerosolización de secreciones desde el paciente durante el procedimiento.

\section{Desconexiones del circuito}

Recomendamos: Evitar desconexiones y en caso de ser necesario, clampear el tubo orotraqueal y poner el ventilador en modo espera antes de desconectar al paciente para evitar la expulsión de aerosoles desde los tubos del ventilador al ambiente. 


\section{Referencias}

1. Surviving Sepsis Campaign: Guidelines on the Management of Critically III Adults with Coronavirus Disease 2019 (COVID-19). Alhazzani W., et al Article in revision and has been accepted for co-publication in the Journals Critical Care Medicine $(\mathrm{CCM})$ and Intensive Care Medicine (ICM)

2. Güldner A, Kiss T, Serpa Neto A, Hemmes SN, Canet J, Spieth PM, et al. Intraoperative protective mechanical ventilation for prevention of postoperative pulmonary complications: a comprehensive review of the role of tidal volume, positive end-expiratory pressure, and lung recruitment maneuvers. Anesthesiology. 2015 Sep;123(3):692-713. https://doi.org/10.1097/ ALN.0000000000000754 PMID:26120769

3. Recomendaciones para el manejo de la insuficiencia respiratoria aguda en pacientes con neumonia por coronavirus v. 1.0 consultado en https:// www.medicina-intensiva.cl/site/ post.php?id=1000322 en Marzo 2020

4. Helwani MA, Saied NN. Intraoperative plateau pressure measurement using modern anesthesia machine ventilators. Can J Anaesth. 2013 Apr;60(4):404-6. https://doi. org/10.1007/s12630-012-98835 PMID:23283735

5. Tomic Jl, Fuenzalida $P$, Jiménez
C, Jara A, Maldonado F, Penna $A$, et al. Recomendación Clínica: Disponibilidad y Uso de Monitorización Perioperatoria. Rev Chil Anest. 2018;47(2):137-44. https://doi.org/10.25237/revchilanestv47n02.12.

6. Recomendaciones para el manejo de pacientes con COVID19 en el perioperatorio. Revista Chilena de Anestesia [Internet]. Asociacion de Medicos Anestesiologos de Chile; 2020 Mar 31;49(2):196-202. Available from: http://dx.doi. org/10.25237/revchilanestv49n02.03

7. https://www.asahq.org/ about-asa/governance-andcommittees/asa-committees/ committee-on-occupationalhealth/coronavirus/clinical-faqs 